Physics

Physics Research Publications

\title{
Proposed design of a josephson diode
}

\author{
J. P. $\mathrm{Hu}$ \\ C. J. $\mathrm{Wu}$
}

X. Dai 


\title{
Proposed Design of a Josephson Diode
}

\author{
Jiangping $\mathrm{Hu},{ }^{1}$ Congjun $\mathrm{Wu},{ }^{2,3}$ and $\mathrm{Xi} \mathrm{Dai}{ }^{4,5}$ \\ ${ }^{1}$ Department of Physics, Purdue University, West Lafayette, Indiana, USA \\ ${ }^{2}$ Kavli Institute for Theoretical Physics, University of California, Santa Barbara, California, USA \\ ${ }^{3}$ Department of Physics, University of California, San Diego, California, USA \\ ${ }^{4}$ Institute of Physics, Chinese Academy of Sciences, Beijing, China \\ ${ }^{5}$ Department of Physics, and Center of Theoretical and Computational Physics, the University of Hong Kong, Hong Kong
}

(Received 1 April 2007; published 9 August 2007)

\begin{abstract}
We propose a new type of Josephson junction formed by two superconductors close to the superconductor-Mott-insulator transition, one of which is doped with holes and the other is doped with electrons. A self-organized Mott-insulating depletion region is formed at the interface between two superconductors, giving rise to an asymmetric response of current to the external voltage. The collective excitations of the depletion region result in a novel phase dynamics that can be measured experimentally in the noise spectrum of the Josephson current.
\end{abstract}

DOI: 10.1103/PhysRevLett.99.067004

PACS numbers: $85.25 .-\mathrm{j}, 73.43 . \mathrm{Nq}$, 74.81.Fa

The Josephson effect is one of the most fundamental effects associated with the superconducting phase, regardless of differences among various superconducting materials. In strongly correlated electron systems, many other quantum phases exist in the vicinity of the superconducting phase, which give rise to many salient features in Josephson junctions. For example, a long range proximity effect in superconducting-antiferromagneticsuperconducting (SAS) junctions was predicted [1] based on the competition between the superconducting and antiferromagnetic phases in high temperature superconductors [2-5]. An abrupt change of the Josephson critical current was predicted in the junction arrays near the superconductor-Mott-insulating phase transitions [6]. In this Letter, we investigate a new design of the Josephson junction by taking advantage of the competition between the superconducting and Mott-insulating phases. The two sides of the junction are hole and electron-doped superconductors, respectively, which are close to the superconductor-Mott-insulator transition. At the interface, a self-organized Mott-insulating region is formed as the tunneling barrier. We dub this junction "Josephson diode." Similarly to the conventional $p-n$ junctions in semiconductor diodes, the depletion region is suppressed by a positive bias voltage and elongated by a negative bias voltage, giving rise to an asymmetric response of the Josephson current to the external voltage. While the geometry of the junction is similar to the $p-n$ junctions in semiconductor diodes, there are fundamental differences. In the Josephson diode, the depletion region (Mottinsulator) is formed completely due to the quantum nature of competing phases. Moreover, the depletion region not only plays a role of tunneling barrier, but also its quantum fluctuation reveals important information of strong correlation effects. The fluctuation of the region boundaries couples to the carrier recombination process which results in an additional phase dynamics that can be measured experimentally in the noise spectrum of the Josephson current. To illustrate the physics of the Josephson diode, we use the Bose-Hubbard model for charged bosons. The long range interaction between bosons is approximated by the self-consistent effective potential $V$ as

$$
\begin{aligned}
H= & -t \sum_{\langle i j\rangle}\left[b^{\dagger}(i) b(j)+\text { H.c. }\right]+\frac{U}{2} \sum_{i} n(i)[n(i)-1] \\
& +\sum_{i}[V(i)-\mu] b^{\dagger}(i) b(i),
\end{aligned}
$$

where $t$ is the hopping integral, $U$ is the short range on-site repulsion. In homogeneous systems, $V(i)$ equals zero due to the charge neutrality maintained by the background charge, and the phase diagram is well known as shown in Fig. 1. Mott-insulating phases appear at commensurate fillings and small values of $t / U$, which can be doped into superconducting phases either by particles or holes. The structure of the Josephson diode is depicted in Fig. 2(a): its left- and right-hand sides correspond to two points $A$ and $B$ in Fig. 1, where the particle densities $\langle n(i)\rangle_{0}=1 \mp \delta$ for $i$ on the left (right) side, respectively. Because of the chemi-

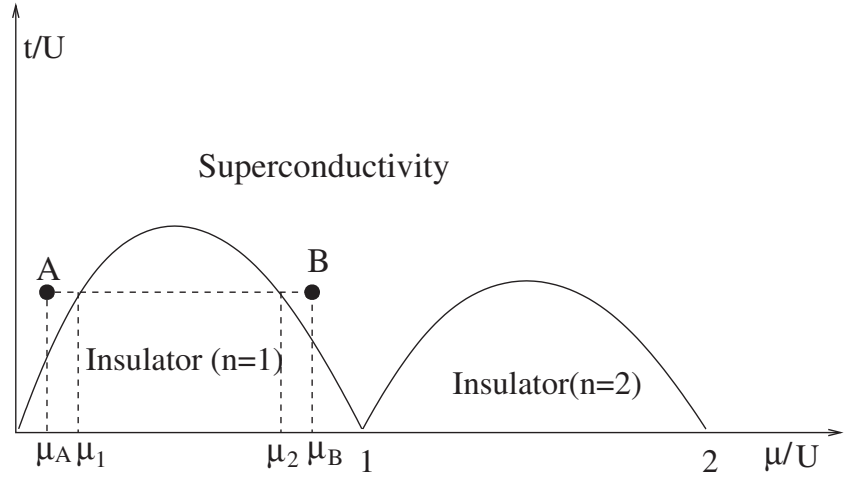

FIG. 1. The mean-field phase diagram of the Bose-Hubbard model. The left (right) side of the junction corresponds to the states at point $\mathrm{A}(\mathrm{B})$. 

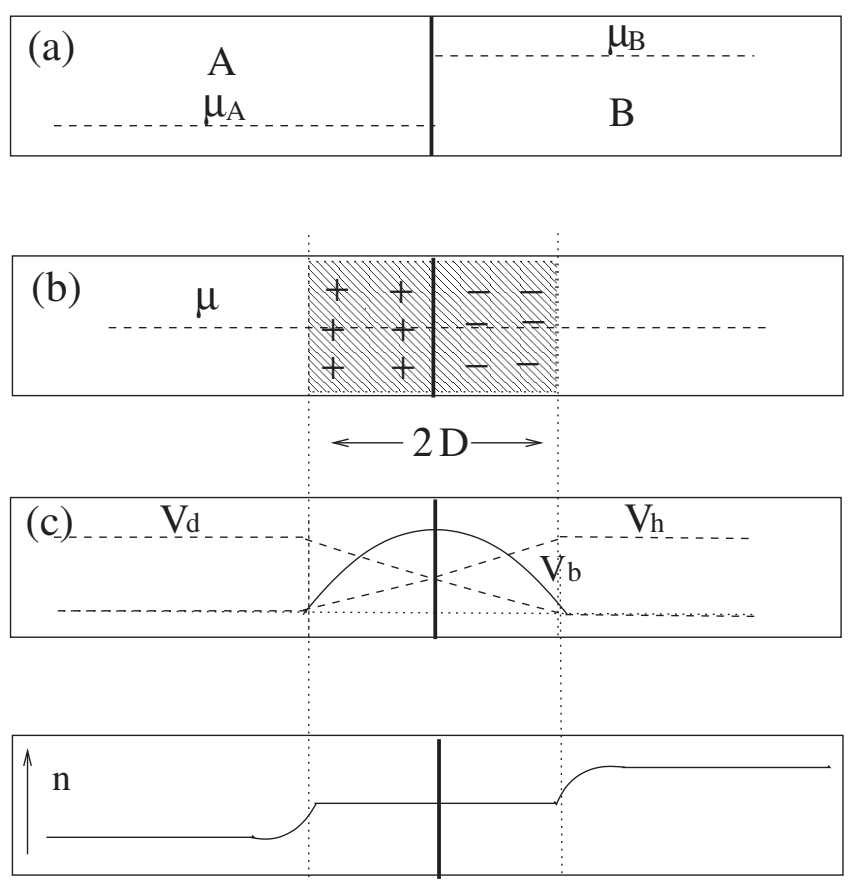

FIG. 2. The sketch of the junction: (a) the spatial distribution of chemical potential before equilibrium is formed, (b) the Mottinsulating depletion region and charge distribution in equilibrium, (c) the potentials $V_{h}$ and $V_{d}$ for the holons and doublons across the junction, respectively.

cal potential imbalance, bosons diffuse from the right to left side. Since a Mott-insulating region interpolates between the hole-doped superconductor $A$ and the particledoped superconductor $B$, it appears across the junction in the real space as the depletion region depicted in Fig. 2(b). We define $\mu_{1(2)}$ as the chemical potentials at the boundary of the Mott-insulating phase depicted in Fig. 1. Inside this region, the density $\langle n(i)\rangle$ is fixed to the commensurate value 1 and the charge neutrality is no longer kept. The resulting internal electric field $E$ gives rise a change of the electric potential $\Delta V$ across the junction to compensate the local chemical potential difference. As a result, the total chemical potential $\mu$ becomes constant. The $E$ field is given by

$$
\nabla E(i)=-\nabla^{2} V(i)=\frac{q}{\epsilon}\left[n(i)-\langle n(i)\rangle_{0}\right],
$$

where $q$ is the charge of bosons, $\epsilon$ is the dielectric constant and the lattice constant is taken as 1 . In the following, we consider the case that $\Delta \mu=\mu_{2}-\mu_{1}$ is much larger than $\mu_{1}-\mu_{A}$ and $\mu_{B}-\mu_{2}$. In this case, most of the charge non-neutral region is the Mott-insulating, and we neglect the contribution to the $E$ field outside the Mott-insulating region. Then $\Delta V$ across the depletion region can be approximated as $\Delta V \approx-\int_{-D}^{D} E(x) d x=\frac{D^{2} q \delta}{\epsilon}=\Delta \mu$, where $2 D$ is the length of the Mott-insulating depletion region, thus

$$
D \approx \sqrt{\frac{\epsilon \Delta \mu}{q \delta}} .
$$

Next we discuss the Josephson diode effect under an external voltage $V_{\mathrm{ex}}$. In the conventional Josephson junction, the coupling term is given by

$$
H_{J}=-J \cos \left(\phi_{r}-\phi_{l}+\frac{q V_{\mathrm{ex}} t}{\hbar}\right),
$$

where the coupling strength $J$ is not sensitive to $V_{\text {ex }}$. However, the situation in the Josephson diode junction is quite different. When $V_{\text {ex }}$ is applied, it not only creates the time dependent phase difference across the junction, but also changes the length of the depletion region. Similarly to the case of semiconductor diodes, the depletion region is suppressed (elongated) in the forward (reversed)-biased junction since the external electric field aligns in the opposite (same) direction of the internal electric field. By a similar reasoning for Eq. (3), we obtain $D$ in the presence of $V_{\mathrm{ex}}$ as $D \approx \sqrt{\epsilon\left(\Delta \mu-V_{\mathrm{ex}}\right) /(q \delta)}$. When $D$ is much longer than the coherent length $\xi$, the Josephson coupling $J$ is proportional to $e^{-\alpha(D / \xi)}$, where $\alpha$ is a dimensionless constant. Therefore, $J$ as a function of $V_{\mathrm{ex}}$ is asymmetric as

$$
\log J\left(V_{\mathrm{ex}}\right) \propto \sqrt{\left(\Delta \mu-V_{\mathrm{ex}}\right) /(q \delta)} .
$$

which is sketched in Fig. 3. This is a natural generalization of the effect of the $p-n$ junction in semiconductors. Please note that when $V_{\mathrm{ex}}$ is close to $\Delta \mu$, the Mott depletion region is almost completely suppressed, then Eq. (5) does not apply.

A major difference between the Josephson and conventional semiconductor diodes is that the charge current is no longer unidirectional in the former case. In the semiconductor diode, the unidirectional charge transport clearly breaks time reversal symmetry, which is consistent with its dissipative nature. In contrast, in the Josephson diode, the dissipationless transport keeps time reversal symmetry. As a result, in the zero bias case, the dc Josephson current can flow either from the $p$ to $n$ side or equivalently well from the $n$ to $p$ side. At the finite bias, the ac Josephson current flows back and forth between the two sides. The asymmetric response to the external voltage lies in the magnitude of the Josephson critical current, which is an effect from the explicit parity breaking in the structure of the Josephson diode junction.

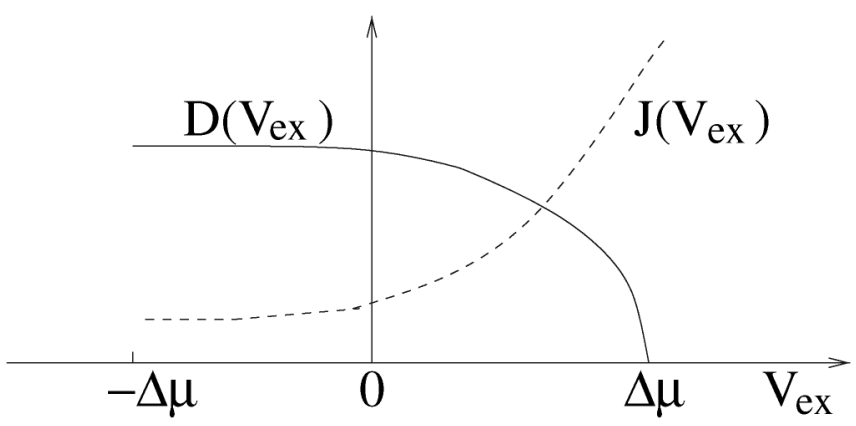

FIG. 3. The length of Mott-insulating depletion region $D$ and the Josephson coupling strength $J$ as a function of the external voltage $V_{\text {ex }}$ (arbitrary unit for the vertical axis). 
In a semiconductor $p$ - $n$ junction, there are the processes of the diffusion and recombination of charge carriers. The analog of such processes also exists in the Josephson diode junction. We introduce the singlon $\left[s^{\dagger}(i)\right]$, holon $\left[a_{h}^{\dagger}(i)\right]$, and doublon $\left[a_{p}^{\dagger}(i)\right]$ operators for the empty, single and double-occupied states on the site $i$, respectively. We limit the Hilbert space on each site to these three states, which is a good assumption for the Bose-Hubbard model when the density is close to 1 . In this case, the holons and doublons are considered as the majority carriers in the left and right sides, respectively. Since each site can only have one such boson, these bosons are hard corelike, thus, namely, they satisfy the following constraint $a_{h}^{\dagger} a_{h}+s^{\dagger} s+a_{p}^{\dagger} a_{p}=1$. We can rewrite the original boson operators in terms of these hard core bosons as $b^{\dagger}=s^{\dagger} a_{h}+a_{p}^{\dagger} s$. The density operator of the original bosons is given by $n=b^{\dagger} b=$ $a_{p}^{\dagger} a_{p}-a_{h}^{\dagger} a_{h}+1$. The hopping term in this representation becomes

$$
\begin{aligned}
H_{t}= & -t \sum_{i j}\left\{\left[a_{h}^{\dagger}(j) a_{h}(i)+a_{p}^{\dagger}(i) a_{p}(j)\right] s^{\dagger}(j) s(i)+\right.\text { H.c. } \\
& \left.+\left[a_{h}(i) a_{p}(j)+a_{p}(i) a_{h}(j)\right] s^{\dagger}(i) s^{\dagger}(j)+\text { H.c. }\right\},
\end{aligned}
$$

where the first two terms describe the hopping processes of holons and doublons and the second two terms describe their recombination and regeneration processes. Correspondingly, the current operator $J_{\text {cur }}$ is given by

$$
\begin{aligned}
J_{\text {cur }}= & i t \sum_{i j}\left\{\left[a_{h}^{\dagger}(i) a_{h}(j)-a_{p}^{\dagger}(j) a_{p}(i)\right] s^{\dagger}(i) s(j)-\text { H.c. }\right\} \\
& -i t \sum_{i j}\left\{\left[a_{h}(i) a_{p}(j)-a_{h}(j) a_{p}(i)\right) s^{\dagger}(j) s^{\dagger}(i)-\text { H.c. }\right\},
\end{aligned}
$$

where the first two terms are the drift currents and the last two terms are recombination and regeneration current.

In order to study the Josephson coupling explicitly in the diode, we take the following general mean-field trial wave function,

$$
\begin{aligned}
\Psi= & \prod_{i}\left\{\cos \theta_{1}(i) s^{\dagger}(i)+e^{i \phi_{2}(i)} \sin \theta_{1}(i)\right. \\
& \left.\times\left[e^{i \phi_{1}(i)} \cos \theta_{2}(i) a_{h}^{\dagger}(i)+e^{-i \phi_{1}(i)} \sin \theta_{2}(i) a_{p}^{\dagger}(i)\right]\right\},
\end{aligned}
$$

which gives the superconducting order parameter as

$$
\left\langle b^{\dagger}(i)\right\rangle=\frac{\sin 2 \theta_{1}(i)}{2} e^{i \phi_{1}(i)}\left[e^{i \phi_{2}(i)} \cos \theta_{2}(i)+e^{-i \phi_{2}(i)} \sin \theta_{2}(i)\right] .
$$

There are two independent phase variables $\phi_{1,2}$ in the above formulas. $\phi_{1}(i)$ is the conventional phase of superconducting order parameter, which is the conjugate variable to the density, namely, $\left[n(i), \phi_{1}(i)\right]=i$. The other variable, $\phi_{2}(i)$ is associated with the quantity $a_{p}^{\dagger} a_{p}+$ $a_{h}^{\dagger} a_{h}=1-s^{\dagger} s$, namely, the density of singlons $n_{s}(i)=$ $s^{\dagger} s$. Thus, we have $\left[n_{s}(i), \phi_{2}(i)\right]=i$. In the conventional
Josephson junction, $\phi_{2}(i)$ can be simply fixed to be zero since the number of singlons can arbitrarily fluctuate. However, the presence of the depletion insulating region changes the above picture. Not only does the depletion region serve as a tunneling barrier, but also its fluctuation leads to additional physics, which is the fluctuation of the number of singlons. Assuming the variables of the wavefunctions in the superconducting states in the left and right sides of the junctions are given by $\left(\phi_{1 l, r}, \phi_{2 l, r}, \theta_{1 l, r}, \theta_{2 l, r}\right)$, we rewrite Eq. (6) as

$$
\begin{aligned}
H_{t}= & J_{1} \cos \left(\Delta \phi_{1}-\Delta \phi_{2}\right)+J_{2} \cos \left(\Delta \phi_{1}+\Delta \phi_{2}\right) \\
& +J_{3} \cos \left(\Delta \phi_{1}-\varphi\right)+J_{4} \cos \left(\Delta \phi_{1}+\varphi\right), \\
\Delta \phi_{i}= & \Delta \phi_{i r}-\Delta \phi_{i l}(i=1,2), \quad \varphi=\phi_{2 l}+\phi_{2 r}, \\
J_{1}= & \sin 2 \theta_{1 l} \sin 2 \theta_{1 r} \cos \theta_{2 l} \cos \theta_{2 r} / 4, \\
J_{2}= & \sin 2 \theta_{1 l} \sin 2 \theta_{1 r} \sin \theta_{2 l} \sin \theta_{2 r} / 4, \\
J_{3}= & \sin 2 \theta_{1 l} \sin 2 \theta_{1 r} \cos \theta_{2 l} \sin \theta_{2 r} / 4, \\
J_{4}= & \sin 2 \theta_{1 l} \sin 2 \theta_{1 r} \sin \theta_{2 l} \cos \theta_{2 r} / 4 .
\end{aligned}
$$

Similarly to the Josephson coupling in multiband superconductors [7-9], Eq. (10) indicates hidden internal dynamics in the junction. There are two observations. The first is that the coupling depends not only on $\Delta \phi_{2}$, but also on $\varphi$, the sum of the phases $\phi_{2 l}$ and $\phi_{2 r}$, reflecting the recombination and regeneration processes. As shown in Fig. 1, holons and doublons are the majority carriers in the left and right sides, respectively, thus $\cos \theta_{2 l} \gg \sin \theta_{2 l}$ and $\sin \theta_{2 r} \gg \cos \theta_{2 r}$ hold. As a result, the $J_{3}$ term describing the recombination and regeneration processes dominates over other terms. The second is that if $\phi_{2}$ is set to 0 , we recover the conventional form of the Josephson coupling energy, i.e., $H=J \cos \left(\Delta \phi_{1}\right)$. Without the insulating region, the number of singlons is not determined in both sides. Therefore, $\varphi$ and $\Delta \phi_{2}$ do not have any dynamics and can be chosen to 0 . However, their dynamics can be created in the presence of the depletion insulating region.

We discuss the fluctuation of the Mott-insulating depletion region. When the system is slightly out of equilibrium, the depletion region has to shrink or expand symmetrically respect to the center of the junction in order to maintain the charge neutrality. This corresponds to the recombination and regeneration processes in the depletion region, which changes the value of $n_{s}^{R}+n_{s}^{L}$, and thus creates the dynamics of $\varphi$. The frequency of this mode can be estimated as follows. The mismatch of chemical potentials in the two sides of the junctions depends on the length of the depletion region as $\Delta \mu_{\text {mis }}=\Delta \mu-\Delta V=\Delta \mu-D^{2} q \delta / \epsilon$. The restoration force on the charge carriers can be approximated as $F=-\Delta \mu_{\text {mis }} /(2 D)=-\Delta x \Delta \mu / D^{2}$, where $\Delta x$ is the small displacement of the boundaries of the depletion region. This mode is essential the plasmon mode with the frequency determined as $\omega_{b}^{2}=q \delta /\left(m^{*} \epsilon\right)$, where $m^{*} \approx$ $1 /(t \delta)$ is the effective mass of the charge carrier. Thus $\omega_{b}$ scales linearly with the doping level $\delta$. 
In general, the fluctuations of the phase $\varphi$ is a diffusion process. Let $\Gamma$ be the decay rate set by the diffusion, which depends on disorder and detailed low energy excitations in the insulating and superconducting regions. The fluctuations of $\varphi$ lead to the following correlation function,

$$
\left\langle e^{i \varphi(t)} e^{-i \varphi(0)}\right\rangle=e^{i \omega_{b} t-\Gamma|t|} .
$$

Therefore, the total current correlation function is

$$
\langle J(t) J(0)\rangle \propto \cos \left(\omega_{b} t\right) e^{-\Gamma|t|},
$$

which gives the noise spectrum of current as

$$
\begin{aligned}
S(\omega) & =\int d t e^{i \omega t}\langle\{J(t)-\langle J(t)\rangle, J(0)-\langle J(0)\rangle\}\rangle \\
& \propto \frac{\Gamma}{\left(\omega-\omega_{b}\right)^{2}+\Gamma^{2}}+\frac{\Gamma}{\left(\omega+\omega_{b}\right)^{2}+\Gamma_{2}^{2}} .
\end{aligned}
$$

Now we briefly discuss the case for neutral bosonic system, say, the $p-n$ junction made by cold bosonic atoms. The Bose-Hubbard model [10] has already been realized in optical lattices experimentally. In such systems, it requires an external potential drop to create a depletion region in an inhomogeneous optical lattice. In this neutral system, there is an additional mode, a sliding mode, associated to the collective excitations of depletion region. For the sliding mode, we can imagine that the system is slightly out of equilibrium by sliding the whole depletion region. Since this mode changes the value $\left(n_{s}^{R}-n_{s}^{L}\right)$, it naturally creates the dynamics of $\Delta \phi_{2}$. In the two band picture, this mode is corresponding to transferring a hole (or doubleon) in the one side to the other side. Since the difference of band energy is given by the Mott gap, $\Delta \mu$ which is proportional to $U$, the mode is expected to have the frequency $\omega_{a}=$ $\Delta \mu$. This mode has the same response in the noise spectrum of the junction as the plasma mode expect their energy difference. A design of the "atomtronic diode" exhibiting asymmetric response to external chemical potential difference was discussed by Seaman et al. [11]. The major difference to our design is that no insulating depletion region exists in their design.

Experimentally, the Bose-Hubbard mode for charged bosons can be realized in Josephson arrays [6]. By choosing the chemical potential configuration discussed above, the Josephson diode should be formed. High $T_{c}$ cuprates are another class of possible systems to realize the Josephson diode. Two different insulating phases exist in cuprates. One is in undoped systems and the other is at the $1 / 8$ doping level $[12,13]$, both of which can be used to create the Josephson diode in principle. A universal asymmetric density between positive and negative bias voltage in STM experiments [14-16] has been observed, which can been explained by considering an insulating competing order at surface [17-20]. This is an important support for the possible realization of the proposed junction, although other alternative explanations $[21,22]$ also exist. The key to create the Josephson diode junction is a spatially selective doping control in cuprates. An in-plane SAS junction has been created by using the spatially selective and reversible doping control techniques in cuprate films [23]. Photodoping techniques can also locally control the doping level [24-26]. These techniques can be used to create two close-by regions with different doping levels with an insulating phase between them. Although it is clear that the Josephson diode in cuprates cannot be described by the simple Bose-Hubbard model, we believe that the physics presented here is still valid. The effective bosonic Hamiltonian in cuprates has to include the spin degree of freedom [3,5,27]. Since we are interested in the charge transport in the Josephson diode, the spin degree of freedom does not have adirect effect on the properties we studied in this Letter. However, it is largely an open question whether any other new properties can be induced by the spin degree of freedom.

We would like to acknowledge the earlier stimulating conversations with S. C. Zhang. J. P. Hu would like to thank the summer school at Boulder, Colorado. J.P. Hu was supported by the National Scientific Foundation under Grant No. Phy-0603759.

[1] E. Demler, A. J. Berlinsky, C. Kallin, G. B. Arnold, and M. R. Beasley, Phys. Rev. Lett. 80, 2917 (1998).

[2] S. C. Zhang, Science 275, 1089 (1997).

[3] S. C. Zhang et al., Phys. Rev. B 60, 13070 (1999).

[4] S. Sachdev, Rev. Mod. Phys. 75, 913 (2003).

[5] E. Demler, W. Hanke, and S. C. Zhang, Rev. Mod. Phys. 76, 909 (2004).

[6] L. I. Glazman and A. I. Larkin, Phys. Rev. Lett. 79, 3736 (1997).

[7] A. J. Leggett, Prog. Theor. Phys. 36, 901 (1966).

[8] D. F. Agterberg, Eugene Demler, and B. Janko, Phys. Rev. B 66, 214507 (2002).

[9] A. J. Leggett, Rev. Mod. Phys. 47, 331 (1975).

[10] M. Greiner et al., Nature (London) 415, 39 (2002).

[11] B. T. Seaman, M. Kraemer, D.Z. Anderson, and M.J. Holland, Phys. Rev. A 75, 023615 (2007).

[12] S.A. Kivelson, E. Fradkin, and V. J. Emery, Nature (London) 393, 550 (1998).

[13] J. Zaanen, Nature (London) 404, 714 (2000).

[14] J. E. Hoffman et al., Science 295, 466 (2002).

[15] T. Hanaguri et al., Nature (London) 430, 1001 (2004).

[16] M. Vershinin et al., Science 303, 1995 (2004).

[17] J. P. Hu and K. J. Seo, Phys. Rev. B 73, 094523 (2006).

[18] DiStasio et al., Phys. Rev. Lett. 64, 2827 (1990).

[19] S. Chakaravarty et al., Phys. Rev. B 63, 094503 (2001).

[20] S. Chakravarty et al., Nature (London) 428, 53 (2004).

[21] W. Rantner et al., Phys. Rev. Lett. 85, 3692 (2000).

[22] P. W. Anderson and N. P. Ong, arXiv:cond-mat/0405518.

[23] O.H. Seongshik et al., Appl. Phys. Lett. 87, 231911 (2005).

[24] A. Gilabert et al., J. Supercond. 13, 1 (2000).

[25] K. Tanabe et al., Phys. Rev. Lett. 72, 1537 (1994).

[26] R. S. Decca et al., Phys. Rev. Lett. 85, 3708 (2000).

[27] W.E. Tsai and S. A. Kivelson, Phys. Rev. B 73, 214510 (2006). 